\title{
ESTRESSE CRÔNICO OCUPACIONAL EM PROFISSIONAIS DA ESTRATÉGIA DE SAÚDE DA FAMÍLIA
}

\author{
Rogério Ferrari ${ }^{1}$ \\ Andréia Araujo ${ }^{2}$ \\ Hingridy Aparecida Rodrigues ${ }^{2}$ \\ Flávia Maria de França ${ }^{3}$ \\ Josiane Magalhães ${ }^{4}$
}

RESUMO: O estresse crônico ocupacional, também conhecido como Síndrome de Burnout, vem afetando, em grande escala, os profissionais de saúde que, dedicados à arte de prevenir e curar doenças, não percebem seu próprio adoecer em consequência do trabalho. O projeto "Burnout em profissionais da Estratégia de Saúde da Família: um diagnóstico da estafa profissional" tem por objetivo a promoção da saúde e a prevenção da síndrome de Burnout nos profissionais de onze Estratégias de Saúde da Família (ESF) da cidade de Cáceres-MT. As atividades desenvolvidas foram divididas em dois momentos: primeiramente, o levantamento de dados nas ESF; posteriormente, a orientação aos profissionais, por meio de palestras acerca da síndrome a fim de constituir, em cada unidade, grupos de trabalho preventivos.

PALAVRAS-CHAVE: Síndrome de Burnout. Estresse no trabalho. Qualidade de vida. Trabalhadores.

\section{Occupational chronic stress in professionals of thefamily health strategy}

\begin{abstract}
Occupational chronic stress, also known as Burnout Syndrome, has affected, in largescale, health professionals who have not realized they were getting ill as a result of their work, although they are dedicated to the art of preventing and curing diseases. The project "Burnout in professionals of the Family Health Strategy: a diagnosis of overwork" (Burnout em profissionais da Estratégia de Saúde da Família: um diagnóstico da estafa profissional) aims to further health and prevention of Burnout syndrome of the elevenprofessionals of Family Health Strategies (FHS), of the city of Cáceres, in the state of Mato Grosso. The activities that were developed were divided into two stages: at first place, it was collected data in the FHS; at second place, it was given guidance to those professionals through lectures about the syndrome aiming to create preventive work groups in each unit.
\end{abstract}

KEYWORDS: Burnout syndrome. Stress at work. Professional exhaustion. Quality of life. Workers.

\footnotetext{
Graduando em Medicina pela Universidade Estácio de Sá (Campus Rio de Janeiro), bolsista FAPEMAT (rgrferrari@gmail.com)

${ }^{2}$ Graduandas em Enfermagem pela Universidade do Estado de Mato Grosso (and.araujo.91@gmail.com) (hingridhi@gmail.com).

${ }_{3}^{3}$ Mestre em Ciências da Saúde pela Universidade de Brasília, professora do Departamento de Enfermagem da Universidade do Estado de Mato Grosso, enfermeira assistencialista do Hospital Regional de Cáceres-MT (flavia43franca@hotmail.com).

${ }^{4}$ Doutora em Educação pela Universidade Estadual Paulista “Júlio de Mesquita Filho", professora adjunta do Departamento de Pedagogia da Universidade do Estado de Mato Grosso, professora dos cursos de graduação e especialização em Gestão em Saúde da Universidade Aberta do Brasil (josimag@gmail.com).
} 
O estresse crônico ocupacional, também conhecido como Síndrome de Burnout, é considerado, atualmente, um grave problema de caráter psicossocial que vem afetando o mundo do trabalho. A síndrome é reconhecida como um risco ocupacional para profissões que envolvem cuidados com saúde, educação e serviços humanos, sendo inclusa em leis brasileiras de auxílio ao trabalhador.

No trabalho, as situações indutoras do estresse são cada vez mais crescentes. Tal preocupação é decorrente da inserção do indivíduo nesse contexto, visto que, além de possibilitar crescimento, transformação, reconhecimento e independência pessoal, o trabalho pode causar também problemas, insatisfação e desinteresse (FRANÇA; FERRARI, 2012).

O Decreto $n^{\circ}$ 3048/99, de 6 de maio de 1996, que dispõe sobre a Regulamentação da Previdência Social, em seu Anexo II, trata dos agentes patogênicos causadores de doenças profissionais (previsto no Art. 20, da Lei ${ }^{\circ}$ 8.213/91), ao se referir aos transtornos mentais e do comportamento relacionados com o trabalho (Grupo V da CID-10); no inciso XII, aponta a "Sensação de Estar Acabado" ("Síndrome de Burnout", "Síndrome do Esgotamento Profissional”) (GIANASI; BORGES, 2009). Porém, o tema ainda é pouco discutido, e, em grande parte, desconhecido, entre os profissionais.

Vivenciando o estresse proveniente do trabalho de forma crônica, o trabalhador precisa desenvolver atitudes que lhe permitam continuar em atividade: uma delas é o não investimento de energia nas tarefas a serem desenvolvidas. Isso leva à destituição de sentido do trabalho e seus resultados passam a não mais importar. Tais sinais são indicativos de que esse trabalhador está vivenciando a Síndrome de Burnout (TELLES; PIMENTA, 2009).

O Burnout pode ser definido como um transtorno adaptativo crônico, associado às demandas e exigências laborais, cujo desenvolvimento é insidioso e, frequentemente, não percebido pelo indivíduo, com sintomatologia múltipla, predominando o cansaço emocional(MOREIRAet al., 2009).

Ainda como definição, Benevides-Pereira (2002) pontua que o Burnout é um processo que se dá em resposta à intensificação do estresse ocupacional, trazendo consigo consequências negativas tanto em nível individual, como profissional, familiar e social.

Como se vê, as conceituações ainda não estão totalmente acordadas entre os pesquisadores. Não há uma única definição específica e fechada para a Síndrome, porém o que é comum a todas elas é a relação com o trabalho (principalmente se este envolve o cuidar, prestar assistência às pessoas com problemas), as consequências exaustivas que traz ao profissional e a mudança que lhe confere no comportamento.

A síndrome de Burnout é caracterizada por três dimensões: Exaustão Emocional (EE), que se refere ao sentimento de esgotamento físico e mental; Envolvimento Pessoal no Trabalho (EPT), caracterizado pela sensação de baixa autoestima, insatisfação com suas atividades e desmotivação; e por fim, a Despersonalização, caracterizada pelo desenvolvimento de atitudes negativas, ausência de sensibilidade, tratamento impessoal e frio dos receptores da assistência. Esta última dimensão é vista por alguns autores como o grande diferencial da Síndrome de Burnout, a característica que lhe é exclusiva. 
A primeira reação do estresse ligado ao trabalho é a sensação de exaustão, esgotamento, sobrecarga física e mental, juntamente com as dificuldades de relacionamento. O ceticismo aparece como tentativa de se proteger da exaustão. As pessoas se tornam mais distantes e frias em relação ao trabalho e aos colegas, uma vez que se sentem mais seguras por assumir uma postura indiferente. Como consequência desse distanciamento, aparece como resultado a ineficiência. Quando os profissionais se tornam ineficientes, perdem a confiança na própria capacidade de fazer diferença e, à medida que perde a autoconfiança, os outros também perdem a confiança neles (LIMA et al., 2007).

Dentre os sintomas que já foram descritos como sendo característicos do Burnout, extraem-se quatro grupos principais: (1) Físicos: fadiga constante e progressiva, distúrbios do sono, dores musculares ou osteomusculares, cefaléias, enxaquecas, perturbações gastrointestinais, imunodeficiência, transtornos cardiovasculares, distúrbios do sistema respiratório, disfunções sexuais e alterações menstruais nas mulheres; (2) Psíquicos: falta de atenção, de concentração, alterações de memória, lentidão do pensamento, sentimento de alienação, sentimento de solidão, impaciência, sentimento de insuficiência, baixa autoestima, labilidade emocional, dificuldade de autoaceitação, astenia, desânimo, disforia, depressão, desconfiança e paranoia; (3) Comportamentais: negligência ou excesso de escrúpulos, irritabilidade, incremento da agressividade, incapacidade para relaxar, dificuldade na aceitação de mudanças, perda de iniciativa, aumento do consumo de substâncias, comportamento de alto risco, suicídio; (4) Defensivos: tendência ao isolamento, sentimento de onipotência, perda do interesse pelo trabalho ou até pelo lazer, absenteísmo, ironia e cinismo (BENEVIDES-PEREIRA, 2002). Todos eles trazem prejuízo ao trabalho que o profissional realiza, resultando em quedas acentuadas da qualidade da assistência prestada.

O desenvolvimento da Síndrome de Burnout envolve vários fatores individuais e laborais sendo, portanto, multicausal, no qual as variáveis socioambientais são coadjuvantes do processo (TRINDADE et al., 2010). Seu surgimento depende de alguns fatores predisponentes, sejam eles organizacionais, de trabalho, sociais e/ou pessoais. A identificação desses preditos é imprescindível no processo de discussão científica sobre Burnout (FRANCA et al., 2012).

Dessa maneira, a saúde mental dos trabalhadores tem se tornado, entre os pesquisadores, um tema constante. Isso porque mudanças no comportamento biopsicossocial do indivíduo geram implicações em sua saúde e, consequentemente, em seu trabalho, ocasionando grandes custos organizacionais (FERRARI; FRANÇA; MAGALHÃES, 2012).

Os profissionais de saúde, geralmente, não percebem seu próprio adoecer em consequência do trabalho, tornando-se um grupo em risco para desenvolvimento da Síndrome de Burnout. Por trabalharem inseridos na comunidade e serem referência de atenção à saúde da população residente em sua área de abrangência, os trabalhadores da Estratégia de Saúde da Família (ESF) precisam assumir inúmeras e diversificadas atribuições. O trabalho nas Unidades de Saúde Pública (USP) é desenvolvido em um ambiente com vários fatores de risco ocupacional, que podem gerar danos à saúde dos trabalhadores e afetar a qualidade da assistência prestada (TRINDADE et al., 2010).

Porém, tais problemas podem ser evitados se os aspectos envolvidos no desenvolvimento da síndrome forem trabalhados corretamente, no âmbito individual, coletivo e organizacional. Existem métodos que tornam possível uma melhoria da qualidade de vida desses profissionais, 
que podem ser vistos como meios de prevenir a Síndrome de Burnout.

Conhecendo os graves problemas que a Síndrome de Burnout pode trazer ao trabalho, e sabendo que ainda são poucas as pesquisas realizadas sobre esse relevante tema no Brasil, foi elaborado o projeto "Burnout em profissionais da Estratégia de Saúde da Família: um diagnóstico da estafa profissional", que vem sendo desenvolvido em dez ESF na cidade de Cáceres-MT, tendo, como público-alvo, médicos, enfermeiros, técnicos de enfermagem, odontólogos e agentes comunitários de saúde dessas instituições. O Burnout possui muitos fatores desencadeadores e inúmeros desdobramentos, precisando ser bem compreendido e estudado para permitir estratégias de intervenção mais eficazes.

O projeto foi criado tendo em vista a importância da valorização do trabalho realizado pelos profissionais da saúde, com o intuito da melhoria da qualidade de vida e bem-estar, condições e ambiente de trabalho. O cuidado permeia o fazer dos profissionais da saúde e, ao mesmo tempo, pode ser causador de danos à saúde do cuidador. Daí a importância desses trabalhadores aprenderem a cuidar de si, evitando e/ou reduzindo os danos desta ocupação e, consequentemente, preservando a saúde, com condições plenas de proporcionar uma assistência adequada aos usuários (RITTER; STUMM; KIRCHER, 2009).

Frente às considerações estabelecidas, as atividades desenvolvidas pelo projeto contribuem para se ter uma visão mais ampla das atitudes e das condições de trabalho desses profissionais com função assistencial, pelo fato de serem elementos indispensáveis ao processo saúde-doença.

\section{MÉTODO}

O projeto está sendo realizado em 10 ESF do município de Cáceres-MT, sendo elas: Vitória Régia, CAIC, Paraíso, Vila Real, Rodeio, Marajoara, Vista Alegre, Cohab Nova, Guanabara e Vila Irene.

As atividades desenvolvidas foram divididas em dois momentos: primeiramente, o levantamento de dados nas ESF e, posteriormente, a orientação aos profissionais por meio de palestras sobre a síndrome, a fim de constituir, em cada unidade, grupos de trabalho preventivos.

Incluíram-se na amostra todos os médicos, enfermeiros, técnicos de enfermagem, odontólogos e agentes comunitários de saúde (ACS) com vínculo formal nessas instituições e dispostos a participar de forma livre, esclarecida e voluntária.

Para a avaliação da Síndrome, no estudo foi utilizado o questionário Maslach Burnout Inventory (MBI). Este método avalia as três dimensões da Síndrome de Burnout: Exaustão Emocional, Envolvimento Pessoal no Trabalho e Despersonalização.

O MBI, composto por 22 perguntas sobre sentimentos e atitudes que englobam os três aspectos fundamentais da Síndrome, deve ser respondido em uma escala que varia de 1 a 5 , sendo: 1 para "Nunca", 2 "Raramente", 3 “Algumas vezes", 4 "Frequentemente" e 5 "Sempre". 
A Exaustão Emocional é avaliada por nove itens, o Envolvimento Pessoal no Trabalho por oito, a Despersonalização por cinco, totalizando os 22 itens. Os escores totais foram calculados para cada uma das três dimensões e pontos de corte foram utilizados para classificar em níveis alto, médio e baixo.

Acombinação dos níveis encontrados nas três dimensões define a Síndrome de Burnout.Assim, quando ocorre associação de altas pontuações em Exaustão Emocional e Despersonalização e a obtenção de baixa pontuação em Envolvimento Pessoal no Trabalho, infere-se que o indivíduo apresenta a Síndrome.

O instrumento elaborado para delinear o perfil sociodemográfico dos profissionais, contém 12 perguntas e levou em contas as variáveis dicotômicas (gênero, duplo vinculo empregatício), de atributos (categoria profissional, grau de escolaridade, carga horária semanal, vínculo empregatício, estado civil, renda mensal, religião) e contínuas (idade, tempo de trabalho na unidade, número de filhos).

As informações dos instrumentos respondidos foram codificadas, digitadas e processadas em planilha Excel.

A equipe executora do projeto é composta por um acadêmico do curso de Medicina (coordenador), duas professoras do Departamento de Enfermagem (orientadoras), dez acadêmicos do curso de Enfermagem da Universidade do Estado de Mato Grosso (UNEMAT), cursando o $7^{\circ}$ e $8^{\circ}$ semestres, sendo dois bolsistas e oito voluntários.

Primeiramente, a equipe do projeto contatou as equipes de Saúde da Família e apresentou-lhes a propostas das atividades a serem desenvolvidas nas respectivas unidades. Posteriormente, cada profissional disposto a participar assinou um termo de consentimento livre e esclarecido.

Encontros têm sido realizados em datas que lhes sejam favoráveis, de modo que cada ESF tem encontros com a equipe do projeto em intervalos que variam de duas vezes por semana a um mês, aproximadamente, de acordo com a disponibilidade da equipe. O primeiro encontro foi dedicado à explanação das atividades propostas e do conceito simplificado da Síndrome, para que houvesse maior aderência ao projeto. Posteriormente, oficinas de grupos, discussões e atividades de palestras foram desenvolvidas periodicamente, em que se buscou discutir soluções para os principais estressores encontrados. Dentre os temas discutidos estavam questões relativas à melhoria da qualidade de vida, enfrentamento do trabalho, prioridades na vida, lazer, atividades saudáveis e apoio social.

O presente estudo foi desenvolvido com anuência dos ESFs e aprovado pelo Comitê de Ética da Universidade do Estado de Mato Grosso, CEP/UNEMAT, em 10 de novembro de 2011 com o Parecer n ${ }^{\circ}$ 205/2011.

\section{RESULTADOS E DISCUSSÃO}

De um total de dez ESF inclusas no projeto, nove já tiveram os dados levantados e as atividades iniciadas, sendo as ESF: COHAB nova, Jardim Guanabara, Jardim Paraíso, Marajoara, Rodeio, 
Vila Real, Vista Alegre, Vitória Régia e Vila Irene.

Por meio da Tabela 1, observa-se que 55 profissionais participaram da pesquisa. Cada ESF contou com cerca de três a oito profissionais participantes. Os ACS formam a grande parte dos profissionais que aderiram à pesquisa, representando $60 \%$, seguidos dos técnicos de enfermagem e enfermeiros, com 16,36\% cada categoria.

Tabela 1- Frequência das ESF e profissionais participantes da pesquisa, Cáceres-MT, 2011.

\begin{tabular}{ccc}
\hline Variável & Frequência & $\%$ \\
\hline ESF: & 8 & 14,54 \\
COHAB Nova & 7 & 12,73 \\
Jardim Guanabara & 7 & 12,73 \\
Jardim Paraíso & 6 & 10,91 \\
Marajoara & 7 & 12,73 \\
Rodeio & 6 & 10,91 \\
Vila Real & 7 & 12,73 \\
Vista Alegre & 4 & 7,27 \\
Vitória Régia & 3 & 5,45 \\
Vila Irene & & \\
& & \\
Profissão: & 33 & 60 \\
ACS & 2 & 3,64 \\
MED & 9 & 16,36 \\
TEC ENF & 1 & 1,82 \\
ODO & 1 & 1,82 \\
THB & 9 & 16,36 \\
ENF & 55 & 100 \\
Total & & \\
\hline
\end{tabular}

Fonte: Elaborada pelos autores.

A maior parte dos pesquisados inserem-se nas seguintes categorias: sexo feminino $(87,27 \%)$, idade entre 36 e 40 anos $(25,45 \%)$, casados $(61,82 \%)$, renda mensal de até três salários mínimos $(69,09 \%)$, carga horária de trabalho de 40 horas semanais $(94,54 \%)$.

A ESF requer dedicação exclusiva dos trabalhadores, exige disponibilidade de 40 horas semanais (oito horas diárias) e ter a ESF como único vínculo empregatício (TRINDADE et al., 2010). Mesmo assim, a pesquisa identificou um percentual de 9,09\% de trabalhadores que possuem outro vínculo empregatício, na maioria das vezes à noite, e o fato pode estar associado à necessidade de aumentar a renda familiar.

Em relação à Síndrome de Burnout, foram identificados três profissionais acometidos. A mesma é indicativa quando se encontra alta Despersonalização, alta Exaustão Emocional e baixo Envolvimento Pessoal no Trabalho. Os acometidos são todos do sexo feminino, sendo os profissionais: uma 
técnica de enfermagem, uma enfermeira e uma agente comunitária de saúde, de distintas ESF.

Cabe ressaltar que, dentre os 55 profissionais pesquisados, existe um número preocupante de profissionais em risco para o desenvolvimento da Síndrome, com alterações significativas em ao menos duas dimensões característica da Síndrome de Burnot.

Diante dos dados apresentados, percebe-se que é necessária atenção no gerenciamento da situação de saúde dos trabalhadores, visto que o Burnout pode apresentar consequências ao processo de trabalho, afetando a qualidade de assistência prestada. A Síndrome gera uma sobrecarga de movimento e tensão ocupacional, sendo necessário monitorar, periodicamente, a saúde mental e física desses trabalhadores, desenvolvendo-se estratégias que possam reorganizar o processo de trabalho diminuindo as fontes de estresse.

O Burnout pode ser evitado, desde que a cultura da organização favoreça a execução de atividades preventivas do estresse crônico, a partir da atuação em equipes multidisciplinares, em uma perspectiva deresgatar as características afetivas contidas no cotidiano de quem cuida (JODAS; HADDAD, 2009).

Após aplicação dos questionários, deu-se início ao segundo momento do projeto, referente à execução de atividades preventivas do Burnout. Inicialmente, foi marcada uma roda de conversa com cada equipe, a fim de esclarecer os conceitos básicos da Síndrome, seus sintomas, possíveis causas e estilos de vida que podem ser adotados para evitar seu aparecimento. As atividades foram desenvolvidas com intuito de configurar um importante objeto de reflexão aos profissionais de saúde, convindo como alerta das causas do desenvolvimento desse tipo de estresse ocupacional de caráter crônico.

Nesse momento, os participantes foram questionados sobre alimentação, padrão de sono, atividade física, atividades prazerosas e religiosidade de cada um, salientando-se que boas estratégias de enfrentamento ao estresse podem ser construídas, como, por exemplo, melhorias nos hábitos de vida.

É fundamental a inclusão de novas práticas aumentem a qualidade de vida dos profissionais, como: hábitos alimentares mais saudáveis; cuidados com a qualidade do sono; prática de exercícios físicos regulares; busca pela qualidade nas relações interpessoais; espaço ocupado pelo trabalho em suas vidas; reorganização do tempo, deixando espaço para o lazer; estabelecimento de prioridades; focalização de aspectos positivos da situação de trabalho (WILTENBURG, 2009).

A elaboração de estratégias organizacionais e individuais de intervenção, como treinamento, capacitação e supervisão dos profissionais dos PSF, é sempre necessária. Isso porque consiste em um modo de minimizar os danos à sua saúde e melhorar a qualidade de vida no trabalho, repercutindo na qualidade dos serviços prestados à população (SILVA; MENEZES, 2008).

Por conseguinte, foram realizados outros encontros, em que assuntos sobre prevenção à Síndrome de Burnout foram abordados. Segue-se fazendo acompanhamento e aconselhamento das equipes para que enfrentem o estresse e não desenvolvam a Síndrome de Burnout. Busca-se, sempre, contribuir para a prevenção de casos da Síndrome por meio da construção do conhecimento e da conscientização acerca da melhoria nas condições de vida e de trabalho, que venha garantir 
ao trabalhador saúde física e mental.

Para que o indivíduo tenha qualidade de vida, é necessário que possua, também, condições de trabalho, entre elas: ambiente adequado, meios para desenvolvê-lo, organização da instituição, alimentação, transporte, relacionamento interpessoal e salário. Algumas determinadas situações vivenciadas no ambiente de trabalho podem levar o indivíduo a um desgaste emocional, contribuindo para o estresse, comprometendo a qualidade de vida do trabalhador e a qualidade de seu trabalho. O desenvolvimento da síndrome depende de uma combinação de fatores individuais, organizacionais e sociais, que, juntos produzem baixa valorização profissional, resultando na Síndrome de Burnot (GOULART et al., 2010).

\section{CONSIDERAÇÕES FINAIS}

As instituições em que o projeto tem sido desenvolvido vêm, gradativamente, incorporando os conhecimentos dos hábitos que podem contribuir para a prevenção da Síndrome de Burnot, podendo, dessa forma, oferecer melhor qualidade no atendimento à população que busca seus serviços de saúde.

As atividades desenvolvidas pelo projeto têm-se mostrado eficientes em levar o conhecimento desse problema aos profissionais e promover melhoria nas condições de trabalho. Pretendem-se, ainda, com o projeto, mais reuniões com as equipes de saúde, visando à melhoria da qualidade de vida e bem-estar de cada um, por meio de conversas, trocas de experiências e dicas que atinjam o âmbito individual e coletivo.

É fundamental o desenvolvimento de medidas que favoreçam o controle do estresse dos profissionais da atenção básica, visto que a Síndrome de Burnout está vinculada a grandes custos organizacionais, por causa do alto absenteísmo, além de queda na produtividade e na qualidade dos serviços prestados. Assim sendo, há necessidade de se avançar na produção de conhecimentos sobre as relações recíprocas entre o trabalho e o bem-estar físico e mental dos trabalhadores.

Por se tratar de um problema social, o conhecimento sobre a Síndrome, suas causas e seus efeitos é imprescindível aos profissionais em risco de acometimento, visto que as consequências afetam diretamente a qualidade da assistência prestada.

Ressalta-se a necessidade de uma maior divulgação sobre a Síndrome de Burnout, já que o desconhecimento das manifestações e causas desse fenômeno faz com que os profissionais de saúde não busquem formas efetivas de prevenção ou intervenção. A divulgação sobre a Síndrome, certamente, contribuirá para isso. 


\section{REFERÊNCIAS}

BENEVIDES-PEREIRA, A. M. Burnout: quando o trabalho ameaça o bem-estar do trabalhador. São Paulo: Casa do Psicólogo, 2002.

FERRARI, R.; FRANÇA, F. M.; MAGALHAES, J. Avaliação da Síndrome de Burnout em profissionais de saúde. Gestão e Saúde, Brasília, v. 3, n. 3, p. 1150-1165, 2012. Disponível em: $<$ http://gestaoesaude.unb.br/index.php/gestaoesaude/article/view/185>. Acesso em: 14 abr. 2012.

FRANÇA, F. M; FERRARI, R. Síndrome de Burnout e os aspectos sociodemográficos em profissionais de enfermagem. Acta Paul. Enferm., São Paulo, v. 25, n. 5, p. 743-748, 2012. Disponível em: < http://www.scielo.br/pdf/ape/v25n5/15.pdf>. Acesso em: 14 abr. 2012.

FRANCA, S. P. S. et al. Preditores da Síndrome de Burnout em enfermeiros de serviços de urgência pré-hospitalar. Acta Paul. Enferm., São Paulo, v. 25, n. 1, p. 68-73, 2012. Disponível em: < http://www.scielo.br/pdf/ape/v25n1/v25n1a12.pdf>. Acesso: 10 abr. 2012.

GIANASI, L. B. S.; BORGES, L. O. Síndrome de Burnout no setor de transporte de Natal. Psic.: Teor. e Pesq., Brasília, v. 25, n. 3, p. 297-305, jul./set. 2009.

GOULART, C. B. et al. Fatores predisponentes da Síndrome de Burnout em trabalhadores de um hospital público de média complexidade. Revista Espaço para a Saúde, Londrina, v. 11, n. 2, p. 48-55, 2010.

JODAS, D. A.; HADDAD, M. C. L. Síndrome de Burnout em trabalhadores de enfermagem de um pronto socorro de hospital universitário. Acta Paul. Enferm., São Paulo, v. 22, n. 2, p. 192-197, 2009. Disponível em: < http://www.scielo.br/pdf/ape/v22n2/a12v22n2.pdf>. Acesso em: 12 abr. 2012.

LIMA, F.D. et al. Síndrome de Burnout em residentes da Universidade Federal de Uberlândia 2004. Rev. Bras. Educ. Med., Rio de Janeiro, v. 31, n. 2, p. 137-146, jan./abr. 2007.

MOREIRA, D. S.; MAGNAGO, R. F.; SAKAE, T. M.; MAGAJEWSKI, F. R. L. Prevalência da Síndrome de Burnout em trabalhadores de enfermagem de um hospital de grande porte da Região Sul do Brasil. Cad. Saúde Pública, Rio de Janeiro, v. 25, n. 7, p.1559-1568, jul. 2009.

RITTER, R. S.; STUMM, E. M. F; KIRCHER, R. M. Análise de Burnout em profissionais de uma unidade de emergência de um hospital geral. Rev. Eletr. Enf., Goiânia, v. 11, n. 2, p. 236248, 2009. Disponível em: < http://www.fen.ufg.br/revista/v11/n2/v11n2a02.htm>. Acesso em: 10 abr. 2012.

SILVA, A.T.C.; MENEZES, P. R. Esgotamento profissional e transtornos mentais comuns em agentes comunitários de saúde. Rev. Saúde Pública, São Paulo, v. 42, n. 5, p. 921-929, 2008. Disponível em: < http://www.scielo.br/pdf/rsp/v42n5/6933.pdf>. Acesso em: 10 abr. 2012.

TELLES, H.; PIMENTA, A. M. C. Síndrome de Burnout em agentes comunitários de saúde e estratégias de enfrentamento. Saúde Soc., São Paulo, v.18, n. 3, p.467-478, jul./set. 2009. 
TRINDADE, L. L. et al. Estresse e Síndrome de Burnout entre trabalhadores da Equipe de Saúde da Família. Acta Paul. Enferm., São Paulo, v. 23, n. 5, p. 684-689, 2010. Disponível em: < http://www.scielo.br/pdf/ape/v23n5/16.pdf>. Acesso em: 10 abr. 2012.

WILTENBURG, D. C. D. Síndrome de Burnout: conhecer para prevenir-se, uma intervenção necessária. Programa de Desenvolvimento da Educação - PDE. Disponível em: <http://www. diaadiaeducacao.pr.gov.br/portals/pde/arquivos/2338-8.pdf>. Acesso em: 14 abr. 2012.

Submetido em 12 de julho de 2012.

Aprovado em 4 de fevereiro de 2013. 